\title{
Time-resolved carrier dynamics and electron-phonon coupling strength in proximized weak ferromagnet-superconductor nanobilayers
}

\author{
T. Taneda, ${ }^{1}$ G. P. Pepe, ${ }^{2}$ L. Parlato, ${ }^{2}$ A. A. Golubov, ${ }^{3}$ and Roman Sobolewski ${ }^{1}$ \\ ${ }^{1}$ Department of Electrical and Computer Engineering and the Laboratory for Laser Energetics, University of Rochester, \\ Rochester, New York 14627-0231, USA \\ ${ }^{2}$ CNR-INFM Coherentia and Dipartimento Scienze Fisiche, Università di Napoli “Federico II," Monte Sant'Angelo, \\ I-80125 Napoli, Italy \\ ${ }^{3}$ Department of Applied Physics, University of Twente, 7500 AE Enschede, The Netherlands
}

(Received 26 February 2007; published 10 May 2007)

\begin{abstract}
We present our femtosecond optical pump-probe studies of proximized ferromagnet-superconductor nanobilayers. The weak ferromagnetic nature of a thin $\mathrm{NiCu}$ film makes it possible to observe the dynamics of the nonequilibrium carriers through the near-surface optical reflectivity change measurements. The subpicosecond biexponential reflectivity decay has been identified as electron-phonon Debye and acoustic phonon relaxation times, and the decay of Debye phonons versus temperature dependence was used to evaluate the electronphonon coupling constants for both the pure $\mathrm{Nb}$ and proximized $\mathrm{Nb} / \mathrm{NiCu}$ heterostructures down to low temperatures. We have also demonstrated that the $\mathrm{NiCu}$ overlay on top of $\mathrm{Nb}$ dramatically reduced the slow, bolometric component of the photoresponse component, making such bilayers attractive for future radiation detector applications.
\end{abstract}

DOI: 10.1103/PhysRevB.75.174507

PACS number(s): 74.25.Gz, 74.70.Ad, 74.78.Fk, 78.47.+p

\section{INTRODUCTION}

Heterogeneous structures formed by a superconductor $(S)$ and a normal metal $(N)$ in contact represent a new and intriguing research field. ${ }^{1,2}$ According to the physics of the proximity effect, ${ }^{3}$ the two materials that form a $S / N$ bilayer influence each other on a spatial scale of the order of the coherence lengths of the two layers (i.e., $\xi_{S}$ and $\xi_{N}$, respectively). In the case of well-investigated bilayers such as $\mathrm{Nb} / \mathrm{Al}, \mathrm{Nb} / \mathrm{Ta}$, and $\mathrm{Ta} / \mathrm{Al}$, their interfaces are highly transparent (metallic), and superconducting correlations are induced in the normal metal since Cooper pairs can penetrate there with a finite lifetime. The $N$ material deposited on top of the $S$ film usually determines the physical properties of the bilayer. If it is very thin, it acts as a thermometer for the electron temperature $T_{e}$ of the underlying $S$ layer, while it is transparent to phonons. ${ }^{4}$ For thicker $N$ overlayers, the carrier and phonon distributions are coupled through suitable boundary conditions at the interface and the interaction is reflected on a spatial variation of the density of states (DoS), directly affecting the electron and phonon lifetimes, as extensively studied in Ref. 2 . Unfortunately, the optical penetration depth $\alpha$ at the 400-900 nm wavelength range (typical for optical experiments) for the materials used most often for $S / N$ bilayers is much smaller than either $\xi_{S}$ or $\xi_{N}$. Thus, the laser probe beam in, e.g., pump-probe experiments can sense only the carrier dynamics of the top layer, far from the interface where the actual proximity effect takes place. A possible solution, presented in this work, is the implementation of a ferromagnet $(F)$ as an $N$ layer, forming $F / S$ bilayers. In such hybrid structures, the superconductor-order parameter decays over a distance of the order of a few nanometers and can be modulated by a proper choice of the magnetic exchange energy, e.g., via varying the thickness of the $F$ film, which can be made comparable to both $\alpha$ and $\xi_{F}$. In general, the coexistence of the $S$ and $F$ states is a very interesting research theme with great potential in quantum electronics (spintronics), ${ }^{5}$ in particular, in phase-dependent superconducting devices. ${ }^{6}$

The aim of this work is to perform time-resolved transient pump-probe spectroscopy measurements to investigate the behavior of proximized $F / S$ nanobilayers below and above the $S$ film critical temperature $T_{c}$. In particular, we are interested in the electron-phonon relaxation time and the role of proximity on the coupling constant in $\mathrm{NiCu} / \mathrm{Nb}$ structures as a function of both the temperature and the $F$ film thickness. Section II presents the basic concepts of the nonequilibrium photoresponse of superconductors and its straightforward physical interpretation. Section III describes the fabrication of our $F / S$ nanobilayer samples and presents the experimental pump-probe-spectroscopy setup used in our time-resolved photoresponse measurements, while Sec. IV shows the results and discusses interpretation and modeling of our experimental data. Finally, Sec. V lists our conclusions.

\section{NONEQUILIBRIUM PHOTORESPONSE OF SUPERCONDUCTORS}

The advent of tunable femtosecond-pulsed lasers opened new possibilities of directly studying fundamental, nonequilibrium electronic processes such as electron-electron $(e-e)$ and electron-phonon $(e-\mathrm{ph})$ interactions in various solidstate materials, including superconductors, where the interactions involve the electron condensate (Cooper pairs), the elementary excitations (quasiparticles), and phonons. ${ }^{7}$ In particular, femtosecond, all-optical, pump-probe spectroscopy has been demonstrated to be very useful in investigating the time-resolved carrier dynamics and the phonon population changes due to the $e$-ph scattering, as well as the other hotelectron effects (e.g., Fermi energy smearing). ${ }^{8}$ From the applied point of view, the nonequilibrium (nonbolometric) pro- 
cesses in superconductors ${ }^{9}$ can lead to the photoresponse with subpicosecond time characteristics, ${ }^{10}$ opening possibilities for the design of extremely energy-sensitive and ultrafast devices for radiation detection. ${ }^{11}$

The time evolution of both the phonon and quasiparticle distribution functions can be described by a system of coupled microscopic kinetic equations. ${ }^{12}$ However, at $T$ $\leqslant T_{c}$ and under weak perturbations, we can use the normalmetal approach and assume that electrons and phonons are described in terms of their energy distributions and assign two different temperatures, $T_{e}$ and $T_{\mathrm{ph}}$, which characterize their relevant subsystems. The nonequilibrium state can therefore be described by a system of two coupled, differential, time-dependent, energy-balance equations ${ }^{13}$ commonly referred to as the two-temperature (2-T) model.

Briefly, absorption of an ultrashort (typically $<1 \mathrm{ps}$ ) laser pulse produces in a superconducting film a nonequilibrium state that involves electron and phonon dynamics. In the initial light-absorption phase, within the optical pulse duration, Cooper pairs are broken, and highly excited electrons thermalize within a few femtoseconds through $e-e$ interaction to re-establish the Fermi distribution with an elevated $T_{e}$, as compared to the sample bath temperature $T_{b}$. Next, these electrons relax, transferring their excess energy to the lattice via $e$-ph interaction with $\tau_{e \text {-ph }}$ of the order of $<1-10 \mathrm{ps}$. In the early stages of relaxation, the electron-Debye-phonon process is the most efficient, while later quasiparticles in a superconductor interact predominantly with acoustic phonons. Finally, phonons cool down by anharmonic decay and/or heat transfer to the substrate. The latter process is called the phonon-escape, and its duration (phonon-escape time $\tau_{\mathrm{es}}$ ) varies from tens of picoseconds to nanoseconds and even microseconds since it depends on the thickness of the superconducting material and the thermal transmissivity of the film-substrate interface.

The 2-T equations, describing the energy balance between the quasiparticles and phonon subsystems, can be written as

$$
\begin{gathered}
C_{e} \frac{d T_{e}}{d t}=\alpha \frac{P_{\mathrm{in}}(t)}{V}-\frac{C_{e}}{\tau_{e-\mathrm{ph}}}\left(T_{e}-T_{\mathrm{ph}}\right)+\frac{C_{\mathrm{ph}}}{\tau_{\mathrm{ph}-e}}\left(T_{\mathrm{ph}}-T_{e}\right), \\
C_{\mathrm{ph}} \frac{d T_{\mathrm{ph}}}{d t}=\frac{C_{e}}{\tau_{e-\mathrm{ph}}}\left(T_{e}-T_{\mathrm{ph}}\right)-\frac{C_{\mathrm{ph}}}{\tau_{\mathrm{ph}-e}}\left(T_{\mathrm{ph}}-T_{e}\right)-\frac{C_{\mathrm{ph}}}{\tau_{e s}}\left(T_{\mathrm{ph}}-T_{b}\right),
\end{gathered}
$$

where $\alpha$ is a radiation absorption coefficient, $V$ is the volume of the sample, $P_{\text {in }}(t)$ is the incident optical power, and $C_{e}$ and $C_{\mathrm{ph}}$ are the heat capacities of the electrons and phonons, respectively. The reverse energy transfer from the phonon subsystem to electrons is characterized by $\tau_{\mathrm{ph}-e}$ $=\tau_{e-\mathrm{ph}}\left(C_{\mathrm{ph}} / C_{e}\right)$. Under low-excitation approximation, the temperature dependences of $C_{e}$ and $C_{\mathrm{ph}}$ can be neglected and the $e$-ph relaxation time can be simplified as $\tau_{e-\mathrm{ph}}=C / g_{e-\mathrm{ph}}$, where $g_{e-\mathrm{ph}}$ is the $e$-ph coupling constant and $1 / C=1 / C_{e}$ $+1 / C_{\mathrm{ph}}$. Experimental determination of $\tau_{e \text {-ph }}$ and $C$ allows one to obtain $g_{e \text {-ph }},{ }^{14}$ which carries fundamental information about the $e$-ph characteristic energy.

The 2-T model is strictly applicable for superconductors at temperatures close to $T_{c}$ (low-perturbation level), when the superconductor energy gap is small and the nonlinear interaction of phonons with Cooper pairs (so-called phonon bottleneck) described by the Rothwarf-Taylor (RT) model ${ }^{15}$ can be ignored. In fact, under the above approximations, the linearized RT equations are formally identical to the 2-T equations. Deviations of the $T_{e}$ and $T_{\mathrm{ph}}$ due to both the Joule power dissipated by the bias current and the absorbed radiation power can also be neglected.

At low temperatures, in addition to the phonon-bottleneck effect, we must take into account the renormalization of the $e$-ph interaction (see, e.g., Ref. 16), due to the presence of so-called shake-off phonons, produced during the initial phase of relaxation of very hot electrons. The above process leads to an additional relaxation channel not considered in the quasiparticle relaxation dynamics described by Eqs. (1) and (2). Highly energetic optical excitation leads to a transition of quasiparticles into an excited, very hot state, corresponding to the energy $E_{0} \gg \Omega_{D}$, where $\Omega_{D}$ is the Debye energy. After the initial, femtosecond-in-duration $e-e$ thermalization, the energy of quasiparticles decreases to the $\Omega_{D}$ level, and the most efficient mechanism for redistribution of energy within the electron subsystem becomes the emission of Debye phonons. The mean free path of those phonons is very small, and they efficiently excite additional electrons (break additional Cooper pairs). The latter process is in the subpicosecond range and lasts until the average electron energy in the avalanche decreases to approximately the superconductor energy gap. At this moment, the quasiparticles are collected near the gap edge, and they relax down by recombination with emission of thermal long-wave acoustic phonons. A microscopic description of this multistage relaxation was proposed by Ovchinnikov and Kresin and by Kozorev et al. ${ }^{17}$

Finally, the 2-T model neglects diffusion of electrons and assumes that the effective temperatures remain uniform within the whole sample. This means that the main mechanism of electron cooling is represented by the $e$-ph transfer rather than electron out-diffusion from the active area. The latter will be applicable in our case, as the thickness of the film is $d=80 \mathrm{~nm}$ and the laser-spot diameter is $\sim 30 \mu \mathrm{m}$. The electron thermal diffusion length in $\mathrm{Nb}$ can be estimated to be $L_{\mathrm{th}}=\left(D \tau_{\mathrm{es}}\right)^{1 / 2} \approx 85 \mathrm{~nm}$, assuming the diffusion coefficient $D=0.3 \mathrm{~cm}^{2} / s$ (Ref. 18) and $\tau_{\mathrm{es}}=4 d /\left(\eta v_{s}\right)=241 \mathrm{ps}$, where the acoustic transmission probability has been assumed to be $\eta=0.25$ and the acoustic velocity is $v_{s}=5.32 \times 10^{3} \mathrm{~m} / \mathrm{s}$. Neglecting diffusion means that electrons and phonons interact mainly within the active volume; consequently, we expect no significant temperature gradients nor temperature dependence of decay times in the range of explored temperatures.

\section{SAMPLE FABRICATION AND EXPERIMENTATION}

Thin films of $\mathrm{Nb}$ and $\mathrm{NiCu}$ alloy were deposited by using two separate dc magnetron-sputtering systems. 60-nm-thick $\mathrm{Nb}$ underlayers were sputtered at a base pressure of 1 $\times 10^{-7}$ Torr on chemically cleaned glass substrates. The sputtering power and the deposition rate were $750 \mathrm{~W}$ and $2.2 \mathrm{~nm} / \mathrm{s}$, respectively. Next, the samples were inserted into the $\mathrm{NiCu}$-dedicated system, where the $\mathrm{Nb}$ surfaces were first 
(a)

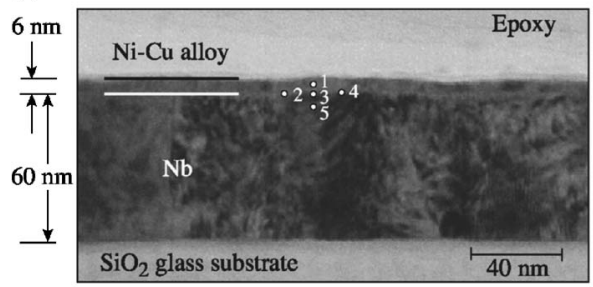

(b) (Atomic \%)

(b)
\begin{tabular}{|c|c|c|c|c|c|}
\hline Position & $\begin{array}{c}\text { NiCu } \\
\text { layer }\end{array}$ & \multicolumn{3}{|c|}{ NiCu/Nb interface } & Nb layer \\
\hline Point \# & 1 & 2 & 3 & 4 & 5 \\
\hline $\mathrm{Ni}$ & 52.7 & 73.2 & 72.9 & 62.6 & 54 \\
\hline $\mathrm{Cu}$ & 47.3 & 26.8 & 27.1 & 37.4 & 46 \\
\hline
\end{tabular}

FIG. 1. A high-resolution TEM cross-sectional image of our sample $\mathrm{C}$. The EDX analysis was performed in five points shown in (a) and the resulting compositions, expressed in terms of atomic percentages of both $\mathrm{Ni}$ and $\mathrm{Cu}$, are listed in (b).

precleaned for $30 \mathrm{~s}$ using ion-beam etching at a rate of about $0.2 \mathrm{~nm} / \mathrm{s}$, and then coated with $\mathrm{Ni}_{0.52} \mathrm{Cu}_{0.48}$ overlayers with thicknesses of $6 \mathrm{~nm}$ (sample C), $12 \mathrm{~nm}$ (sample D), and $21 \mathrm{~nm}$ (sample E) at a rate of $1.5 \mathrm{~nm} / \mathrm{s}$ using a sputtering power density of $10 \mathrm{~W} / \mathrm{cm}^{2}$. Independently, two control films, one of pure $\mathrm{Nb}$ (sample A) and the other of pure $\mathrm{Ni}_{0.52} \mathrm{Cu}_{0.48}$ (sample B) with thicknesses of $60 \mathrm{~nm}$ and $50 \mathrm{~nm}$, respectively, were deposited for reference experiments. Low-temperature magnetization tests performed for plain $\mathrm{Ni}_{0.52} \mathrm{Cu}_{0.48}$ films showed a Curie temperature of $30 \mathrm{~K}$ and a spontaneous moment per atom $\mu_{0}=0.06 \mu \mathrm{B} /$ atom at $T=4.2 \mathrm{~K}$

Figure 1(a) shows a transmission electron microscope (TEM) picture of sample $\mathrm{C}$. We note that the $\mathrm{Nb}$ film is polycrystalline, while the $\mathrm{NiCu}$ is either amorphous or polycrystalline with a grain size $<1 \mathrm{~nm}$. The $\mathrm{NiCu} / \mathrm{Nb}$ interface is almost atomically flat and very sharp. We expect that samples D and $\mathrm{E}$ have $F / S$ interfaces of the same quality. The energy-dispersive $\mathrm{x}$-ray (EDX) spectroscopy analysis confirmed that the composition of the deposited $\mathrm{NiCu}$ alloy was very close to the source target [point 1 in Fig. 1(a)], although we observed a somewhat higher concentration of $\mathrm{Ni}$ close to the $F / S$ interface [points 2, 3, and 4 in Fig. 1(a)] and a partial diffusion of the $\mathrm{NiCu}$ on the $\mathrm{Nb}$ side [point 5 in Fig. 1(a)]. The detailed compositions at indicated points, expressed in terms of atomic percentages of both $\mathrm{Ni}$ and $\mathrm{Cu}$, are listed in Fig. 1(b).

Femtosecond pump-probe-spectroscopy experiments were performed using a commercial, mode-locked Ti:sapphire laser, which produced $100 \mathrm{fs}$ pulses at an $810 \mathrm{~nm}$ wavelength and a $76 \mathrm{MHz}$ repetition rate. The pump and probe beams were focused onto the sample, down $<30 \mu \mathrm{m}$ in diameter, and cross polarized to eliminate coherent artifacts caused by the interference of the two beams. The pump-probe average power ratio was set at 10:1, after initial characterization was performed at different pump powers to be sure of the absence of any trivial thermal effects. ${ }^{19}$ The pump power of $30 \mathrm{~mW}$ (average power: $400 \mathrm{pJ}$ energy per pulse) was selected for the bulk of our experiments as a value low enough to minimize optical heating while ensuring good signal-to-noise ra-
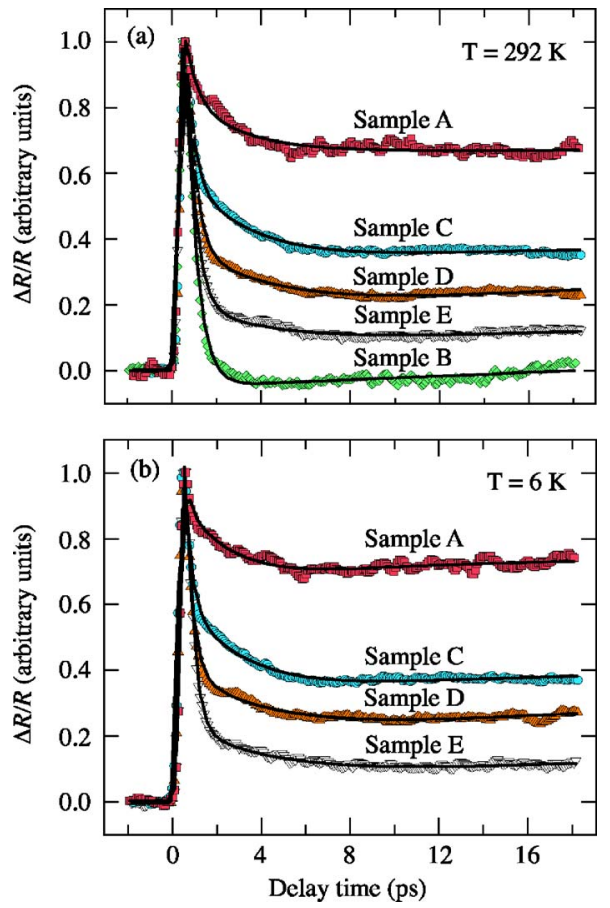

FIG. 2. (Color online) Normalized reflectivity change $\Delta R / R$ as a function of the time delay, measured for all of our tested samples. (a) Room-temperature data; (b) $T=6 \mathrm{~K}$.

tio. We estimate that under $30 \mathrm{~mW}$ of the pump power, the temperature of the overall sample surface did not increase more than $0.3-0.4 \mathrm{~K}$.

The $F / S$ samples were mounted on a cold finger (directly facing the finger's $\mathrm{Cu}$ surface with a 5-mm-diam hole) inside a temperature-controlled, liquid-helium, continuous-flow optical cryostat operating down to $<5 \mathrm{~K}$. The $\mathrm{NiCu} / \mathrm{Nb}$ superconducting transition, measured by the constant-current method, was $T_{c}=8.9 \mathrm{~K}$, which reflected the $T_{c}$ of the bottom (shunting) $\mathrm{Nb}$ layer and, indeed, corresponded to the $T_{c}$ of our pure $\mathrm{Nb}$ film (sample $\mathrm{A}$ ). The optical penetration depth at $810 \mathrm{~nm}$ wavelength for the $\mathrm{Ni}_{0.52} \mathrm{Cu}_{0.48}$ film (sample B) was established to be $\alpha=10.6 \mathrm{~nm}$ by separately measuring the optical transmissivities of a collection of $\mathrm{NiCu}$ films with various thicknesses.

\section{EXPERIMENTAL RESULTS AND DISCUSSION}

Pump-probe studies were performed in reflection mode in a temperature range between 4.8 and $11 \mathrm{~K}$ and at room temperature $(292 \mathrm{~K})$. Figure 2 presents the normalized reflectivity changes $\Delta R / R$ as a function of time, measured for samples A-E at $T=292 \mathrm{~K}$ [Fig. 2(a)] and $T=6 \mathrm{~K}$ [Fig. 2(b)]. The wave forms shown in Fig. 2(b) are very representative for all low-temperature measurements. In general, irrespective of temperature, all $\Delta R / R$ curves were very similar. Within our experimental, 20-ps-wide time window, the photoresponse signal for each sample exhibited an ultrafast rise, limited by the pump-pulse width, followed by a biexponential decay with two characteristic times-the initial, subpicosecond-in-duration fast relaxation time $\tau_{\text {fast }}$ and the 

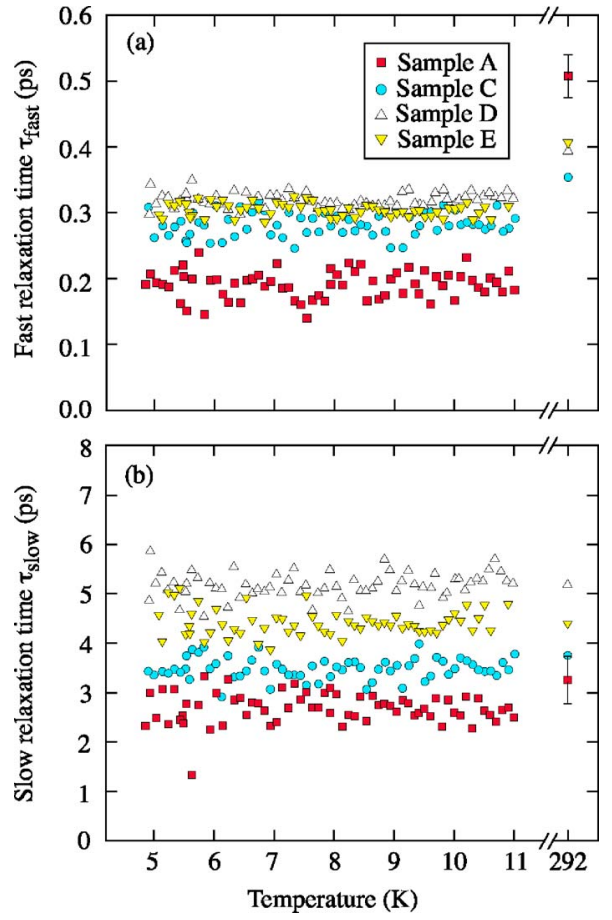

FIG. 3. (Color online) Temperature dependences of the (a) fast and (b) slow relaxation times extracted from the $\Delta R / R$ data obtained for all of our $\mathrm{NiCu} / \mathrm{Nb}$ bilayers and for the pure $\mathrm{Nb}$ film.

second, slower relaxation time $\tau_{\text {slow }}$ of the order of a few picoseconds. $^{20}$ Finally, after the initial relaxation, we observed a plateau that decayed on a hundreds-of-picosecond time scale and clearly represented the bolometric phonon escape. Since the signal amplitude depended on the pumppulse width and amplitude, we normalized amplitudes of all measured wave forms to avoid any dependence on spurious surface effects.

Using a simple fitting formula with two exponential dependencies for the decay part of the signal (thin solid lines in Fig. 2), we extracted the $\tau_{\text {fast }}$ and $\tau_{\text {slow }}$ values from all of our experimental data sets and plotted them as a function of temperature in Figs. 3(a) and 3(b), respectively. We note that our fits are extremely good. In Fig. 3(a), the low-temperature $\tau_{\text {fast }}$ varies overall between $\sim 0.18$ ps (sample A) to $\sim 0.32 \mathrm{ps}$ (samples D and E), but for each sample, the dependence is rather flat and no special features can be observed. The room-temperature $\tau_{\text {fast }}$ values are measurably longer with $\tau_{\text {fast }}$ for pure $\mathrm{Nb}$ (sample A) being more than twice that of the averaged low-temperature value. The $\tau_{\text {slow }}$ time, shown in Fig. 3(b), varies from $\sim 2.5 \mathrm{ps}$ (sample A) to $\sim 5.2 \mathrm{ps}$ (sample D) in the entire temperature range. In the $\tau_{\text {slow }}(T)$ case, we observe a rich structure with peak magnitudes well above our experimental error level, but we were unable to associate those features with any expected temperature dependences such as, e.g., divergence corresponding to the $T_{c}$ of the $F / S$ bilayer.

The focus in this paper was placed on the $\tau_{\text {fast }}$ and $\tau_{\text {slow }}$ processes, since we associate them with the dynamics of the $e$-ph relaxation, where $\tau_{\text {fast }}$ and $\tau_{\text {slow }}$ represent scattering on the Debye and acoustic phonons, respectively. Simultaneously, we note that the plateau level depends very strongly on the thickness of the $\mathrm{NiCu}$ overlayer. The plateau is the most pronounced for the pure Nb film [sample A, Fig. 2] and is completely missing in the pure $\mathrm{NiCu}$ case [sample $\mathrm{B}$, Fig. 2(a)]. The latter behavior shows that, while in pure Nb films the photon escape mechanism appears to be conducted through the $\mathrm{Nb}$-glass interface, in $\mathrm{NiCu}$ films, it is dominated by the ultrafast anharmonic phonon decay. Consequently, in our $F / S$ bilayers, the optical response is weighted by the ratio of the thicknesses of the $\mathrm{NiCu}$ and $\mathrm{Nb}$ layers within the optical penetration depth. Obviously, the strongly suppressed bolometric plateau in $F / S$ bilayers makes them very attractive for fast optical superconducting detectors.

The absence of any discontinuities in the electronacoustic-phonon relaxation $\tau_{\text {slow }}(T)$ dependence near $T_{c}$ deserves some comments, since one might expect a divergence when approaching the transition from the superconducting to normal state. We need to stress that our result is in agreement with experiments reported in Ref. 14, where no divergence at $T_{c}$ was observed in pump-probe studies of $\mathrm{Nb}$. The apparent reason is that the jump in the electron heat capacity at $T_{c}$ in $\mathrm{Nb}$ is not very pronounced. ${ }^{21}$ In addition, according to a semiquantitative approach proposed by Zaitlin, ${ }^{22}$ the proximity effect in $S / N$ bilayers further smears out any such jump with respect to the pure superconductor case. The latter fact is known from a theory of multiband (or anisotropic) superconductivity. ${ }^{23}$ Mathematically, the $S / N$ (or $S / F$ ) proximity bilayers are equivalent to a two-band superconductor: in the proximity effect, the two order parameters are coupled in a real space, while in the multiband system, they are coupled in the momentum space.

We also stress that absorption of high-energy photons (for $\mathrm{Nb}, \varepsilon \geqslant E_{F}$ ) leads to unbalanced populations of the hole- and electron-like branches of the system DoS. As a consequence, the scattering time, which describes the relaxation to equilibrium, renormalizes the electron scattering time as $\tau_{Q}$ $=\tau_{e}\left(T_{e} / \Delta\right)$, where $\tau_{e}$ is the relaxation time and $\Delta$ the superconducting energy gap. In the case of $\mathrm{Nb}$, the ratio $k_{B} T_{e} / \Delta$ is expected to be much larger than 1 , and hence $\tau_{Q} \gg \tau_{e}$. This means that gap opening should occur at relaxation times much longer that those studied in our pump-probe experiments.

According to the proximity model for the $F / S$ bilayer proposed in Ref. 24 and on the basis of our earlier investigations of $\mathrm{Ni}_{0.50} \mathrm{Cu}_{0.50} / \mathrm{Nb}$ bilayers, ${ }^{25}$ we have evaluated $\xi_{S}$ $=12 \mathrm{~nm}$ and $\xi_{F}=24 \mathrm{~nm}$. Moreover, we numerically calculated the energy dependence of the local DoS at any position with respect to the $F / S$ interface, as well as on both sides of the bilayer. The knowledge of DoS (Fig. 4) was used to obtain the $C_{e}$ values for our bilayers and $\mathrm{Nb}$ at the temperature $T=6.5 \mathrm{~K}$ (selected as, approximately, the midpoint of our experimental data) using the general (integral form) expression of Ref. 26. The results are listed in Table I. Simultaneously, the lattice contribution to $C$ was calculated according to the basic $C_{\mathrm{ph}}=\beta T^{3}$ law, where the coefficient $\beta$ in the bilayer was obtained by averaging $\beta(\mathrm{Nb})$ and $\beta(\mathrm{NiCu})$ (Ref. 27) with the layer thicknesses normalized to the optical penetration depth as weights. Both the values of $\beta$ and the resulting $C_{\mathrm{ph}}$ at $6.5 \mathrm{~K}$ are listed in Table $\mathrm{I}$.

The $\Delta R / R$ transients shown in Fig. 2 allowed us to estimate the peak temperatures for both the electrons and 


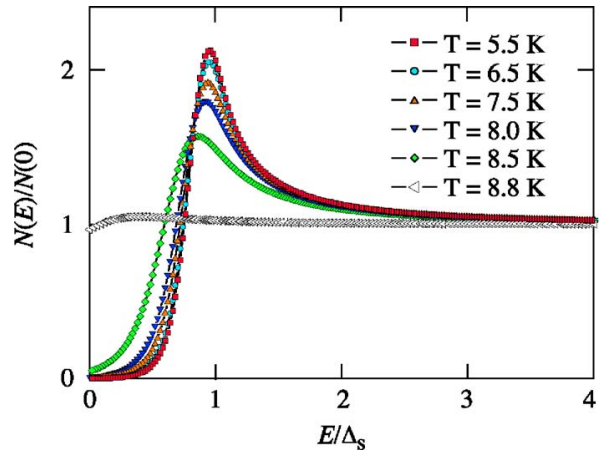

FIG. 4. (Color online) Density-of-states functions versus the normalized energy, calculated based on the $S / F$ proximity theory (Ref. 15) for sample $\mathrm{C}$ for several temperatures. The function corresponding to $T=8.8 \mathrm{~K}$ represents the normal state.

phonons by assuming that the $T_{e} / T_{\mathrm{ph}}$ ratio was proportional to the $\Delta R / R$ amplitude-to-plateau ratio. The data for $\mathrm{Nb}$ were analyzed by following Ref. 7 for metals, and we obtained $T_{e}=450 \mathrm{~K}$ and $T_{\mathrm{ph}}=311 \mathrm{~K}$, leading to the average temperature $T_{\mathrm{av}}=380 \mathrm{~K}$ and, hence, $T_{\mathrm{av}} / \tau_{\text {fast }}=9 \times 10^{14} \mathrm{~K} / \mathrm{s}$. The latter value was needed to calculate the spectral dependence of the second momentum of the Eliashberg function $\lambda_{\mathrm{eff}}\left\langle\omega^{2}\right\rangle=2 \int_{0}^{\infty} d \omega \alpha^{2} F(\omega) \omega \cong\left(\pi k_{B} / 3 h\right)\left(T_{\mathrm{av}} / \tau_{e-\mathrm{ph}}\right)$, equal to $324 \mathrm{meV}^{2}$, and it turned out to be in agreement with previous reports. ${ }^{28}$ The situation is clearly more complicated for the proximized bilayers, since our $\Delta R / R$ experiments clearly show that the $T_{e} / T_{\mathrm{ph}}$ ratio quickly increases with the increase of the $\mathrm{NiCu}$ overlayer thickness. For sample E, which exhibits the lowest bolometric plateau and, thus, represents the most promising $F / S$ composition toward detection experiments, the estimated $T_{\mathrm{av}}$ is as high as $\sim 1500 \mathrm{~K}$ and, consequently, $\lambda_{\text {eff }}\left\langle\omega^{2}\right\rangle \sim 1300 \mathrm{meV}$, a value much larger than that found for ordinary metals. ${ }^{28}$ Clearly, a thin $\mathrm{NiCu}$ overlayer on top of $\mathrm{Nb}$ plays a very effective active role, leading to the very high transient values of $T_{e}$ and resulting in ultrafast photoresponse. This latter feature is extremely important toward achievement of ultrafast photodetectors with wellcontrolled (engineered), superconducting-state electronic response times.

The estimation of $g_{e-p h}$ using our experimental values of $\tau_{\text {fast }}$ as $\tau_{e-\mathrm{ph}}$ has been successfully obtained for the room-

TABLE I. The basic parameters of our tested $F / S$ samples extracted from our experiments, collected for $T=6.5 \mathrm{~K}$. The values of $\Delta_{S F-F_{\text {side }}}$ have been obtained by considering the lowest values at which $N(E) / N(0)=1$.

\begin{tabular}{lcccc}
\hline \hline Sample & $\mathrm{A}$ & $\mathrm{C}$ & $\mathrm{D}$ & $\mathrm{E}$ \\
\hline$d_{F} / \xi_{F}$ & 0 & 0.25 & 0.50 & 0.875 \\
$\Delta_{S F-F_{\text {side }}}(\mathrm{meV})$ & 1.35 & 1.17 & 1.11 & 0.85 \\
$\beta\left(\mathrm{J} / \mathrm{K} \mathrm{m}^{3}\right)$ & 10.5 & 6.6 & 3.8 & 3.8 \\
$C_{e}\left(\mathrm{~J} / \mathrm{K} \mathrm{m}^{3}\right)$ & $8.7 \times 10^{3}$ & $9.5 \times 10^{3}$ & $9.7 \times 10^{3}$ & $9.4 \times 10^{3}$ \\
$C_{\mathrm{ph}}\left(\mathrm{J} / \mathrm{K} \mathrm{m}^{3}\right)$ & $2.9 \times 10^{3}$ & $1.8 \times 10^{3}$ & $1.0 \times 10^{3}$ & $1.0 \times 10^{3}$ \\
$\tau_{e-\mathrm{ph}}=\tau_{\text {fast }}(\mathrm{ps})$ & 0.18 & 0.27 & 0.32 & 0.31 \\
$g_{e-\mathrm{ph}}\left(\mathrm{W} / \mathrm{K} \mathrm{m}^{3}\right)$ & $1.2 \times 10^{16}$ & $5.6 \times 10^{15}$ & $2.8 \times 10^{15}$ & $2.9 \times 10^{15}$ \\
$\lambda_{\text {eff }}\left\langle\omega^{2}\right\rangle\left(\mathrm{meV}^{2}\right)$ & $2 T_{e}$ & $1.3 T_{e}$ & $1.1 T_{e}$ & $1.2 T_{e}$ \\
\hline \hline
\end{tabular}

temperature data. On the other hand, the value for $g_{e-\mathrm{ph}}$ (see Table I) is in good agreement with the prediction in Ref. 29 at $T=4.2 \mathrm{~K}$. The change in $g_{e-p h}$ observed as a function of the $\mathrm{NiCu}$ thickness clearly points to a role of the proximity effect through the DoS modification for $F / S$ bilayers and, consequently, its influence on $C_{e}$.

The initial stage of relaxation, represented by $\tau_{\text {fast }}$, corresponds (according to formalism in Ref. 17) to quasiparticle interaction with Debye phonons and the relaxation time can be approximated as $\tau \cong \hbar / \lambda \Omega_{D}$, where $\lambda$ is the electronphonon constant. The experimental determination of the electron fast relaxation time through the reflectivity measurements allowed us to estimate the values of $\lambda$ in our bilayers at low temperatures. Assuming $\Omega_{D} \quad(\mathrm{Nb})=276 \mathrm{~K}$ $=22.1 \mathrm{meV}$ and $\Omega_{D}\left(\mathrm{Ni}_{0.48} \mathrm{Cu}_{0.52}\right)=321 \mathrm{~K}=26.8 \mathrm{meV}$ (Ref. 30), we obtain $\lambda_{\mathrm{Nb}}=1.03$ and $\lambda_{\mathrm{Nb} / \mathrm{NiCu}}=0.50$ in the two limiting cases of samples $\mathrm{A}$ and $\mathrm{D}$, respectively. The value obtained for $\mathrm{Nb}$ is in quite good agreement with the similar determinations reported in Ref. 28. It is clear that the presence of a thin proximized $F$ layer on top of the superconducting film produced a measurable difference in the $e$-ph relaxation processes, resulting in a higher effective electron temperature as demonstrated by the $\Delta R / R$ measurements. We know that $\lambda$ should depend mainly on the phonon mode spectrum and the $e$-ph matrix element, but, nevertheless, the proximity effect plays a significant role by introducing another scattering channel as suggested by the similarity with the two-gap model. ${ }^{20}$

\section{CONCLUSIONS}

We have performed comprehensive femtosecond optical pump-probe measurements of proximized $F / S$ bilayers formed by a ferromagnet $\left(\mathrm{Ni}_{0.52} \mathrm{Cu}_{0.48}\right)$ and a superconductor $(\mathrm{Nb})$. The weak ferromagnetic nature of $\mathrm{NiCu}$ makes it possible to observe the nonequilibrium electron dynamics through the near surface via time-resolved $\Delta R / R$ measurements. The fast component of the $\Delta R / R$ decay was identified as the Debye $e$-ph relaxation time, and its temperature dependence was used to evaluate the $e$-ph coupling constant $g_{e-\mathrm{ph}}$ in our proximized $F / S$ heterostructures by calculating the DoS functions for the tested bilayers. In the case of the pure $\mathrm{Nb}$ and for the thick $(21 \mathrm{~nm}) F$ bilayer, we derived the value of the second momentum of the Eliashberg phonon spectrum $\lambda_{\text {eff }}\left\langle\omega^{2}\right\rangle$ from our experiments. In the case of $\mathrm{Nb}$, the agreement with available data in literature is satisfactory also in terms of the $e$-ph constant $\lambda$, thus confirming the validity of our approach. The results for a $\mathrm{NiCu}(21 \mathrm{~nm}) / \mathrm{Nb}$ $(60 \mathrm{~nm})$ sample indicate a very strong reduction of the slow phonon-escape mechanism, due to the presence of a ferromagnetic layer on top of the surface of a superconductor and the apparent ultrafast, anharmonic acoustic phonon relaxation within the $F / S$ interface. The latter feature can be successfully used for future development of custom-made, $F / S$-type ultrafast superconducting optical detectors.

\section{ACKNOWLEDGMENTS}

The work in Rochester was supported by the U.S. AFOSR 
Grant No. FA9550-06-1-0348, with additional support provided by the BBN Technologies and the NYSTAR grant to the University of Rochester CAT-EIS Center. The work of Napoli was partially supported by M.I.U.R. under the Grant
PRIN2006 "Effetti quantistici macroscopici e dispositivi superconduttori" and by EU under the FoNE Project "SpinDependent Transport and Electronic Correlations in Nanostructures."
${ }^{1}$ A. A. Golubov, E. P. Houwman, J. G. Gijsbertsen, J. Flokstra, H. Rogalla, J. B. le Grand, and P. A. de Korte, Phys. Rev. B 49, 12953 (1994).

${ }^{2}$ E. Esposito, G. Pepe, and U. Scotti di Uccio, J. Supercond. 7, 951 (1994).

${ }^{3}$ A. Barone and G. Paternó, Physics and Applications of the Josephson Effect (Wiley, New York, 1982).

${ }^{4}$ S. D. Brorson, A. Kazeroonian, J. S. Moodera, D. W. Face, T. K. Cheng, E. P. Ippen, M. S. Dresselhaus, and G. Dresselhaus, Phys. Rev. Lett. 64, 2172 (1990).

${ }^{5}$ G. Blatter, V. B. Geshkenbein, and L. B. Ioffe, Phys. Rev. B 63, 174511 (2001).

${ }^{6}$ A. I. Buzdin, Rev. Mod. Phys. 77, 935 (2005); A. A. Golubov, M. Yu. Kupriyanov, and E. Il'ichev, ibid. 76, 411 (2004); F. S. Bergeret, A. F. Volkov, and K. B. Efetov, ibid. 77, 1321 (2005).

${ }^{7}$ P. B. Allen, Phys. Rev. Lett. 59, 1460 (1987).

${ }^{8}$ R. W. Schoenlein, W. Z. Lin, J. G. Fujimoto, and G. L. Eesley, Phys. Rev. Lett. 58, 1680 (1987).

${ }^{9}$ D. N. Langenberg and A. I. Larkin, Nonequilibrium Superconductivity, Modern Problems in Condensed Matter Sciences Vol. 12 (North-Holland, Amsterdam, 1986).

${ }^{10}$ M. Lindgren, M. Currie, C. Williams, T. Y. Hsiang, P. M. Fauchet, R. Sobolewski, S. H. Moffat, R. A. Hughes, J. S. Preston, and F. A. Hegmann, Appl. Phys. Lett. 74, 853 (1999).

${ }^{11}$ A. D. Semenov, G. N. Gol'tsman, and R. Sobolewski, Supercond. Sci. Technol. 15, R1 (2002).

${ }^{12}$ G. M. Éliashberg, Sov. Phys. JETP 11, 696 (1960).

${ }^{13}$ M. L. Kaganov, I. M. Lifshitz, and L. V. Tanatarov, Sov. Phys. JETP 4, 173 (1957).

${ }^{14}$ M. Mihailidi, Q. Xing, K. M. Yoo, and R. R. Alfano, in Ultrafast Lasers Probe Phenomena in Semiconductors and Superconductors, edited by R. R. Alfano (SPIE, Bellingham, WA, 1992), Vol. 1677, p. 108.

${ }^{15}$ A. Rothwarf and B. N. Taylor, Phys. Rev. Lett. 19, 27 (1967).

${ }^{16}$ M. Mihailidi, Q. Xing, K. M. Yoo, and R. R. Alfano, Phys. Rev.
B 49, 3207 (1988).

${ }^{17}$ Yu. N. Ovchinnikov and V. Z. Kresin, Phys. Rev. B 58, 12416 (1998); A. G. Kozorezov, A. F. Volkov, J. K. Wigmore, A. Peacock, A. Poelaert, and R. den Hartog, ibid. 61, 11807 (2000).

${ }^{18}$ R. Cristiano, E. Esposito, L. Frunzio, C. Nappi, G. Ammendola, L. Parlato, G. Pepe, H. Kraus, and P. Walko, J. Appl. Phys. 86, 4580 (1999).

${ }^{19}$ G. P. Pepe, L. Parlato, R. Latempa, P. D'Acunto, N. Marrocco, C. De Lisio, C. Altucci, G. Peluso, A. Barone, T. Taneda, and R. Sobolewski, IEEE Trans. Appl. Supercond. 15, 2942 (2005).

${ }^{20}$ Y. Xu, M. Khafizov, L. Satrapinsky, P. Kus, A. Placenik, and R. Sobolewski, Phys. Rev. Lett. 91, 197004 (2003).

${ }^{21}$ A. T. Hirshfeld, H. A. Leupold, and H. A. Boorse, Phys. Rev. 127, 1501 (1962).

${ }^{22}$ M. P. Zaitlin, Phys. Rev. B 25, 5729 (1982).

${ }^{23}$ W. L. McMillian, Phys. Rev. 175, 537 (1968); A. A. Golubov and I. I. Mazin, Phys. Rev. B 55, 15146 (1997).

${ }^{24}$ A. A. Golubov, M. Y. Kupriyanov, and Y. V. Fominov, JETP Lett. 75, 190 (2002).

${ }^{25}$ G. P. Pepe, R. Latempa, L. Parlato, A. Ruotolo, G. Ausanio, G. Peluso, A. Barone, A. A. Golubov, Y. V. Fominov, and M. Y. Kupriyanov, Phys. Rev. B 73, 054506 (2006).

${ }^{26}$ C. Kittel, Introduction to Solid State Physics, 7th ed. (Wiley, New York, 1996).

${ }^{27}$ Y. S. Touloukian and E. H. Buyco, Specific Heat: Metallic Elements and Alloys, Thermophysical Properties of Matter Vol. 4 (IFI/Plenum, New York, 1970), and references therein.

${ }^{28}$ S. D. Brorson, A. Kazeroonian, J. S. Moodera, D. W. Face, T. K. Cheng, E. P. Ippen, M. S. Dresselhaus, and G. Dresselhaus, Phys. Rev. Lett. 64, 2172 (1990).

${ }^{29}$ K. M. Yoo, X. M. Zhao, M. Siddique, R. R. Alfano, D. P. Osterman, M. Radparvar, and J. Cunniff, Appl. Phys. Lett. 56, 1908 (1990).

${ }^{30}$ K. P. Gupta, C. H. Cheng, and P. A. Beck, Phys. Rev. 133, A203 (1964). 